\title{
The mossflora of Camiguin Island, Philippines and their floristic relations to some adjacent islands in the archipelago
}

\author{
Virgilio C. Linis \\ Philippine National Herbarium, Botany Division, National Museum \\ P. Burgos Ave. cor. Taft Ave., Ermita, Manila 1000 Philippines.
}

\begin{abstract}
The moss flora of Camiguin Island, based on a 2007 expedition conducted by the author, totaled 129 species in 66 genera and 29 families. This represents $17.36 \%$ of the 743 moss species and $26.83 \%$ of the 246 genera of mosses reported for the Philippines (Linis \& Tan 2008). Of these, one taxon, Aerobryopsis cochlearifolia Dix., is reported for the first time in the archipelago. Floristically, Camiguin moss flora is identified more with Mindoro and Luzon within the Philippine archipelago. Likewise, the presence of moss taxa such as Glossadelphus hermaphroditus Fleisch. and Orthomnion javense (Fleisch.) T. Kop. highlights the role of Mindanao in enriching the moss flora of Camiguin Island. Finally, the importance of remaining forests on the island as a proposed protected area is discussed.
\end{abstract}

\section{Introduction}

Camiguin (Fig. 1) is a small and compact volcanic island province about $54 \mathrm{~km}$ southeast of Bohol Island and $10 \mathrm{~km}$ north of Misamis Oriental province in the island of Mindanao. With a land area measuring $291.87 \mathrm{sq} . \mathrm{km}$, it lies in the waters of Bohol Sea with geographic coordinates of $124^{\circ} 35^{\prime}$ to $124^{\circ} 5^{\prime}$ east longitude and $9^{\circ} 5^{\prime}$ to $9^{\circ} 20^{\prime}$ north latitude (DENR 1998).

The island is part of the northern extreme of Camiguin-Balatocan Range, a volcanic system formed by constructive Quaternary volcanism in Mindanao Island. Despite this geologic connection, however, no historic land link existed between these two islands (Abad 1949). Camiguin has several volcanoes with Mt. Timpoong (1,525 masl) and Mt. Hibok-hibok (1,086 masl) as two of its highest peaks. The latter is classified as an active volcano and is considered a high risk area (PHILVOCS 1990).

\section{Study Areas and Methods}

On two trips in May 2007, I documented Camiguin's diversity of mosses. During the first expedition, I joined a team of biodiversity experts from Mindanao State University-Iligan Institute of Technology (MSU-IIT) between $19^{\text {th }}$ and $22^{\text {nd }}$ May 2007 
and surveyed Mt. Timpoong with particular emphasis on the northern slope of the mountain. Afterwards, I returned to the island to survey Mt. Hibok-hibok between $28^{\text {th }}$ and $31^{\text {th }}$ May 2007, concentrating on its north-eastern slope. During these two expeditions, pertinent information about the species habitat, substrates, altitude and other relevant data were recorded. Preliminary identifications, at least to generic level, were done in the field. Final identification was done in the Philippine National Herbarium (PNH), subsequently.

I used Kroeber's \% of similarity formula to compare and analyze the floristic affinity of mosses found in Camiguin Island and the first four largest islands within the Philippines: Luzon, Mindoro, Palawan and Mindanao (van Balgooy 1971). Other islands in the archipelago were excluded from this analysis as the bryological information available is inadequate.

Altogether, about 350 specimens of mosses were collected. The voucher specimens of these collections were deposited at $\mathrm{PNH}$ with some duplicate specimens sent to the herbarium of MSU-IIT, Iligan City, Lanao del Norte province in Mindanao.

\section{Results and Discussion}

Moss diversity: only a few scattered publications mention the mosses of Camiguin Island. Bartram (1939) cited only 20 species (Table 1) while Tan \& Iwatsuki (1991) listed 66 taxa when they published their Philippine moss checklist more than fifty years later. Based on this study I accepted 129 species in 66 genera of mosses for Camiguin Island representing $17.36 \%$ of the 743 moss species and $26.83 \%$ of the total 246 moss genera reported for the Philippines (Linis \& Tan 2007).

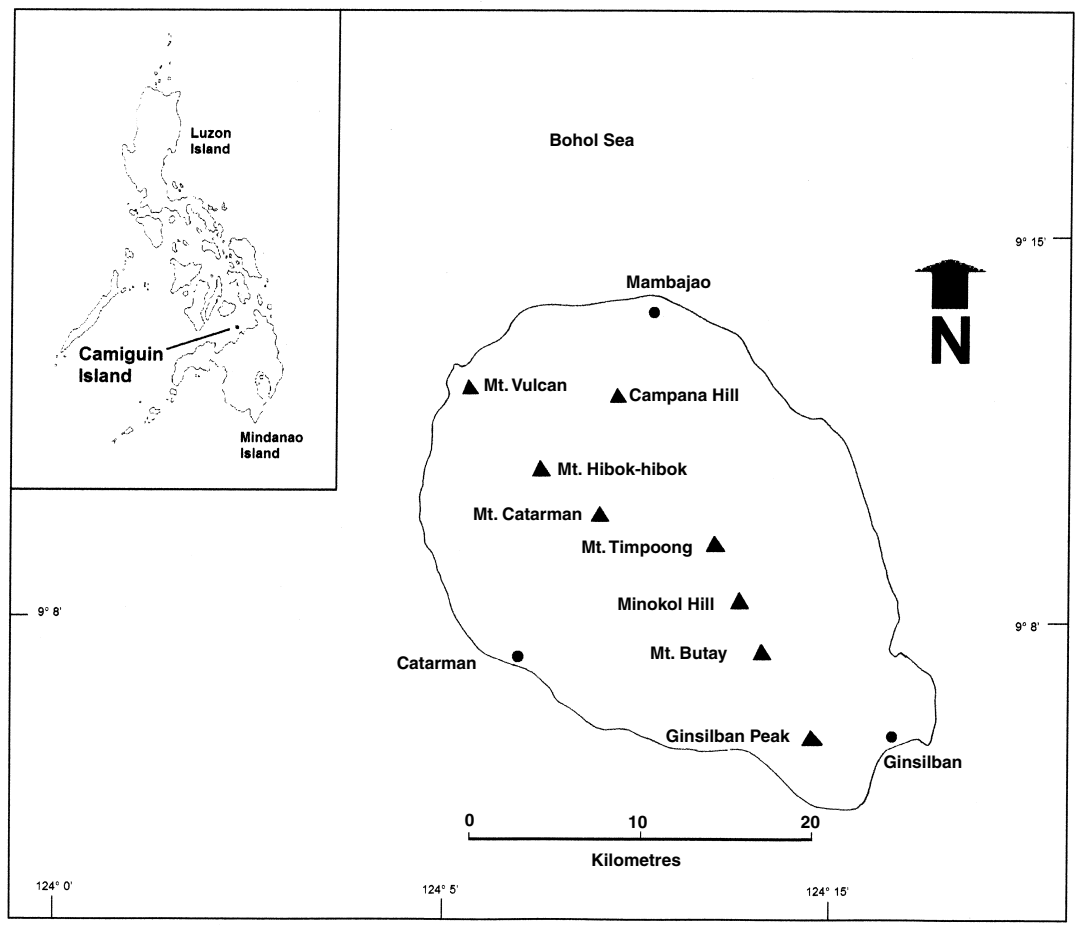

Fig .1. Map of Camiguin Island, Philippines. 
Table 1. Comparison of the moss flora of Camiguin Island as reported in this paper with that of Bartram (1939) and Tan \& Iwatsuki (1991).

Families

1. Polytrichaceae

2. Diphysciaceae

3. Funariaceae

4. Fissidentaceae

5. Ditrichaceae

6. Bruchiaceae

7. Dicranaceae

8. Leucobryaceae

9. Calymperaceae

10. Pottiaceae

11. Orthotrichaceae

12. Bartramiaceae

13. Bryaceae

14. Mniaceae

15. Rhizogoniaceae

16. Spiridentaceae

17. Hypnodendraceae

18. Racopilaceae

19. Ptychomniaceae

20. Hypopterygiaceae

21. Daltoniaceae

22. Pilotrichaceae

23. Thuidiaceae

24. Meteoriaceae

25. Hypnaceae

26. Entodontaceae

27. Sematophyllaceae

28. Pterobryaceae

29. Neckeraceae

Total
2007 Expedition

Genera Species

Tan \& Iwatsuki (1991)

Genera

1

1

11

15

1

1

4

4

2

2

1

1

2

1

1

1

1

1

1

2

2

3

4

6

2

10

4
3
66

3

66

Worth mentioning among these collections are the 65 new records for the island (Appendix 1, marked with asterisk). Eight taxa are extensions of range within the Philippines: Campylopodium medium; Clastrobryum caudatum; Cyathophorum hookerianum; Diphyscium rupestre; Distichophyllum osterwaldii; Glossadelphus hermaphroditus; Pterobryopsis gedehensis and, Syrrhopodon prolifer. 
From the new collections, one species, Aerobryopsis cochlearifolia Dix., proves to be a new record for the Philippine moss flora (Fig. 2). Previously this species was found only in Indochina and was known only from two collections in Laos and Thailand (Noguchi 1976).

In general, the moss diversity of Camiguin Island is highest in mid-elevation forests from 600 to 1500 masl. At these elevations, mosses were observed to be most abundant in wetter forests found on the east-facing slopes of the volcanic peaks and in riverine forests. Such forests support hygrophytic moss genera like Orthomnion, Cyathophorum, Calyptrochaeta, Distichophyllum, Hypnodendron and Garovaglia. Below these elevations, however, the number and diversity of mosses decreases due to the prevalence of grasslands and cultivated areas. In these semi-dry and anthropogenic habitats, moss genera such as Barbula, Bryum, Hyophila, Garckea, Campylopodium and Calymperes thrive.

Floristic affinity: the Camiguin moss flora is most similar to that of Mindoro followed by Luzon (Table 2). Many of the taxa not shared by Mindoro and Camiguin are contributed by those taxa present in Mindoro and neighboring Luzon but absent from the rest of the islands within the Philippine archipelago. Other taxa not shared by Mindoro and Camiguin include those known only from Mindoro such as Rhacocarpus alpinus (Wright.) Par., Dicranoloma daymannianum Bartr. and Distichophyllum noguchianum Tan (Mindoro's sole endemic). The remaining taxa are likely to be undercollected and may well occur elsewhere in the Philippines.
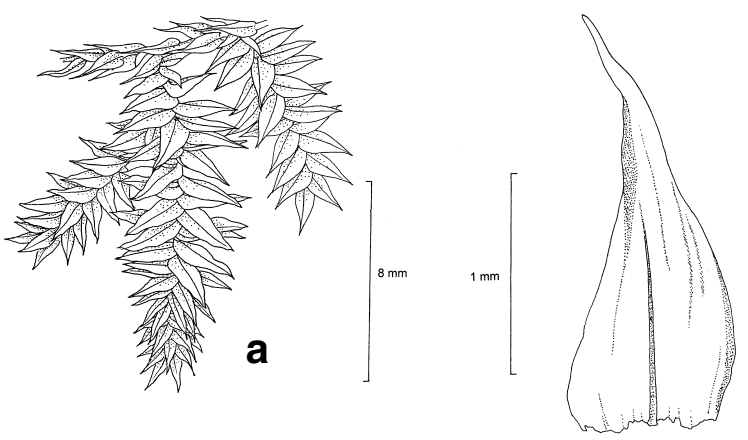

b
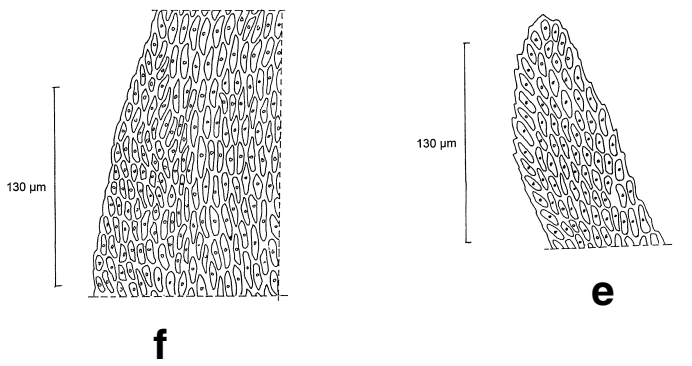
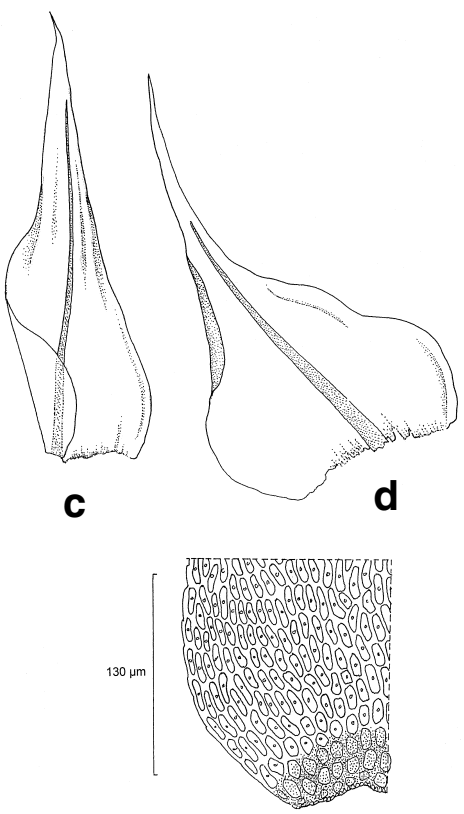

g

Fig. 2. Aerobryopsis cochlearifolia Dix. a, part of the plant; b, c, branch-leaves; d, stem leaf; e, apex of branch leaf; $\mathbf{f}$, cells from middle of branch leaf; $\mathbf{g}$, cells from base of branch leaf. 
Table 2. Kroeber's percentage of taxic similarity $(\% \mathrm{~K})$ between the moss floras of Camiguin, Luzon, Mindoro, Palawan and Mindanao. The numbers are derived from the current work plus previous reports by Bartram (1939), Linis (2004, 2007), Linis \& Tan (2005), Tan (1996), Tan \& Iwatsuki (1991), Tan \& Mandia (2001), Tan et al. (2000).

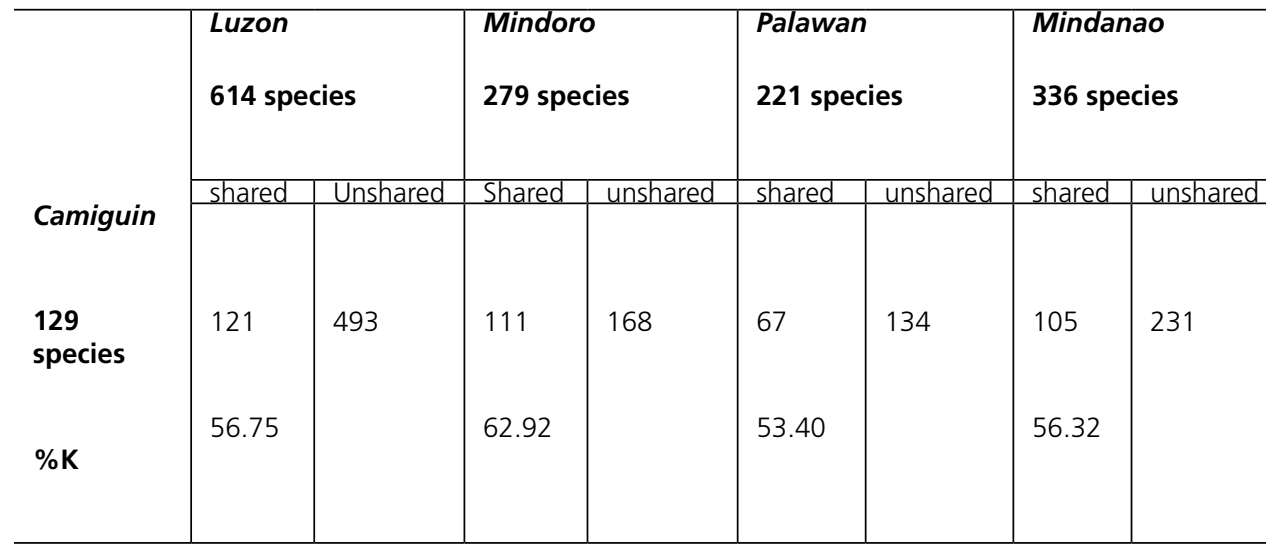

${ }^{*}$ Kroeber's $\%$ of similarity $=C(A+B) / 2 A B \times 100$

$A=$ the number of taxa present in the first area under comparison

$B=$ the number of taxa present in the second area under comparison

$\mathrm{C}=$ the number of common taxa in both $\mathrm{A} \& \mathrm{~B}$ areas.

Most of the taxa not common to Luzon and Camiguin are those species restricted to higher altitudes, over 1800 masl, mostly in the Northern Cordillera of Luzon (Atrichum crispulum Schimp. ex Besch., Chrysocladium flammeum (Mitt.) M.Fleisch., Fabronia curvirostris Dozy \& Molk., Heterophyllium affine (Hook.) M.Fleisch., Leptohymenium tenue (Hook.) Schwägr., Neckera crenulata Harv. and Timmiella anomala (Bruch \& Schimp.) Limpr.) or island endemics (Calyptothecium squarrosulum Nog. \& B.C. Tan, Chaetomitrium perarmatum Broth., Jaegerina williamsii E.B. Bartram and Stereophyllum linisii Enroth \& B.C. Tan). Remaining unshared taxa are likely to be an artifact of under-collecting. It is my opinion that long-distance dispersal is the most likely explanation for this strong relationship between Luzon and Camiguin, as many mosses are dispersed by wind-borne spores. Another possibility is that moss taxa have 'island hopped' along the intervening islands, finally reaching Camiguin. In view of the large number of shared taxa involved between Luzon and Camiguin it is most likely that many of these taxa may eventually be found in the intervening islands.

Taxa not common to Camiguin and Mindanao islands include South Malesian and Australasian genera such as Bryobrothera, Dawsonia, Ectropotheciopsis, Leskeodon and Meiotheciella that appear to have reached only Mindanao in the Philippines. At least two species of mosses: Glossadelphus hermaphroditus Fleisch. and Orthomnion javense (Fleisch.) T.Kop., formerly known in the Philippines only from Mindanao, were found in Camiguin during the first 2007 expedition. This could indicate that dispersal from Mindanao to Camiguin is occurring, but it is more probable, given the islands are separated by c. $10 \mathrm{~km}$, that floristic differences between Camiguin and Mindanao are mainly due to under-collection of moss taxa in northern Mindanao (Tan et al. 2000). 
Finally, Palawan Island which supports semi-dry and seasonally deciduous type of rain forests has a moss flora least similar to that of Camiguin. This is not surprising as Palawan supports a handful of xeric or drought-tolerant moss taxa not found in the rest of the Philippine archipelago. Other Palawan taxa, such as Clastrobryum asperrimum (Dix.) Tan and Horikawaea tjibodensis (M.Fleisch.) M.C.Ji \& Enroth, represent South and West Malesian taxa that have apparently failed to reach other islands of the Philippines. Undercollection may also have a role to play in the distribution patterns reported here.

\section{Acknowledgments}

I am grateful to Mindanao State University- Iligan Institute of Technology, Iligan City for inviting me to participate in their expedition to Camiguin Island in 2007. To Dr. Emelina Mandia of De La Salle University-Manila and Dr. Benito C. Tan of Singapore Botanical Gardens who kindly read the draft and offered substantial suggestions for improvement. To the Biology Department of the University of the Philippines-Manila for lending me their photomicroscope. Lastly to Yong Kien Tai of University of Malaya who encouraged me to write this paper.

\section{References}

Abad LT (1949) A report on the geology and volcanism of Camiguin Island. Bureau of Mines: Manila.

Bartram EB (1939) Mosses of the Philippines. Philippine Journal of Science 68: 1-423.

Ji CM \& Enroth J (2008) Identity of Neckera tjibodensis. Journal of Bryology 28 (3): 167-169.

Department of Environment and Natural Resources (1998) Ecological profile of Camiguin Island. DENR Region 10: Cagayan de Oro City.

Linis VC (2004) A checklist of mosses in Mt. Arayat, Pampanga. Journal of Tropical Biology 3: 2-6.

Linis VC (2009) Biogeography of Mindoro mosses. Blumea 54 (1-3): 290-296.

Linis VC \& Tan BC (2010) The biodiversity and biogeography of mosses of Zambales Mountain Range and Mt. Arayat, Luzon Island, Philippines. In: Proceedings of the $6^{\text {th }}$ International Flora Malesiana Symposium, Los Baños, Laguna Philippines (in press).

Linis VC \& Tan BC (2008) Progress of studies on phytogeography and biodiversity of Philippine moss flora from 1991 to 2006. pp. 13-22. In: Mohamed H, Baki BB, Nasrulhaq-Boyce A \& Lee PKY (eds) Bryology in the New Millennium. (University of Malaya: Kuala Lumpur)

Noguchi A (1976) A taxonomic revision of the family Meteoriaceae of Asia. Journal of the Hattori Botanical Laboratory 41: 231-357.

Tan BC (1996) Biogeography of Palawan mosses. Australian Systematic Botany 9: 193-203.

Tan BC \& Iwatsuki Z (1991) A new annotated Philippine moss checklist. Harvard Papers Botany 3: 1-64.

Tan BC, Lubos L \& Schwarz U (2000) New and biogeographically noteworthy records of Philippine mosses from Mindanao Island. Tropical Biology 18: 27-37.

Tan BC \& Mandia EH (2001) New and noteworthy records of mosses from Mindoro, Philippines and their biogeographical implications. Gardens Bulletin of Singapore 53: 315-322.

Tupas MH (1952) The activity of Hibok-hibok volcano since January 1952. Bureau of Mines: Cagayan de Oro City.

van Balgooy, MJ (1971) Plant geography of the Pacific, as based on the distribution of Phanerogram genera, Blumea suppl. no. 6. Leiden. 


\section{Appendix. Updated checklist of Camiguin mosses and their intra-Philippine distribution.}

* New records added from the 2007 expedition.

1. Acanthorrhynchium papillatum (Harv.) M.Fleisch. - widespread

Mt. Hibok-hibok, 600m elev., on tree roots and lower tree trunk; Residual lowland forest, May 31, 2007, V.C. Linis 2779-07.

2. Acroporium diminutum (Brid.) M.Fleisch. - widespread Mt. Timpoong, $1350 \mathrm{~m}$ elev., on dead tree trunk; Montane forest, May 21, 2007, V.C. Linis 256307.

3. *A. hermaphroditum (Müll.Hal.) M.Fleisch. - Luzon and Mindanao

Mt. Timpoong, 1350 m elev., on tree branch; Montane forest, May 21, 2007, V.C. Linis 2554-07.

4. $\quad *$ A. ramicola (Hampe) Broth. - Mindanao

Mt. Timpoong, 1530 m elev., on tree branch; Montane forest, May 21, 2007, V.C. Linis 2578-07.

5. *A. secundum (Reinw. \& Hornsch.) M.Fleisch. - Luzon, Mindoro, Palawan \& Western Mindanao Mt. Timpoong, $1530 \mathrm{~m}$ elev., on tree trunk; Montane forest, May 21, 2007, V.C. Linis 2593-07.

6. $\quad *$ A. sigmatodontium (Müll.Hal.) M.Fleisch. - Luzon, Mindoro and Panay

Danao, Mambajao (midslope of Mt. Timpoong), $1000 \mathrm{~m}$ elev., on tree trunk; Transitional lowland forest, May 19, 2007, V.C. Linis 2498-07. Mt. Hibok-hibok, 900 m elev., on tree trunk; Transitional lowland forest, May 30, 2007, V.C. Linis 2691-07.

7. A. strepsiphyllum (Mont.) B.C.Tan var. strepsiphyllum - Luzon and Mindanao

Danao, Mambajao (midslope of Mt. Timpoong), $1000 \mathrm{~m}$ elev., on tree trunk; Transitional lowland forest, May 19, 2007, V.C. Linis 2493-07. Mt. Timpoong, $1350 \mathrm{~m}$ elev., on tree branch; Montane forest, May 21, 2007, V.C. Linis 2560-07. , Mt. Hibok-hibok, 900 m elev., on tree trunk; Transitional lowland forest, May 30, 2007, V.C. Linis 2686-07.

8. *Aerobryopsis cochlearifolia Dix. - new Philippine record

Danao, Mambajao (midslope of Mt. Timpoong), 1000 m elev., on tree trunk near creek; Transitional lowlandforest, May 20, 2007, V.C. Linis 2622-07.

9. A. wallichii (Brid.) M.Fleisch. - widespread

Brgy. Pandan, Mambajao (midslope of Mt. Timpoong), $680 \mathrm{~m}$ elev., on tree trunk; Lowland forest, May 19, 2007, V.C. Linis 2442-07. Brgy. Pandan, Mambajao (midslope of Mt. Timpoong), $680 \mathrm{~m}$ elev., on lower tree branches; Lowland forest, May 19, 2007, V.C. Linis 2444-07. Brgy. Pandan, Mambajao (midslope of Mt. Timpoong), $850 \mathrm{~m}$ elev., on tree branch; Transitional lowland forest, May 19, 2007, V.C. Linis 2474-07. Danao, Mambajao (midslope of Mt. Timpoong), $1000 \mathrm{~m}$ elev., on tree branch; Transitional lowland forest, May 19, 2007, V.C. Linis 2497-07. Mt. Timpoong, 1350 m elev., on tree branch; Montane forest, May 21, 2007, V.C. Linis 2568-07. Mt. Hibok-hibok, 320 m elev., on tree trunk; Residual lowland forest, May 29, 2007, V.C. Linis 2635-07. Mt. Hibok-hibok, $700 \mathrm{~m}$ elev., on tree branch; Transitional lowland forest, May 29, 2007, V.C. Linis 2667-07. Mt. Hibok-hibok, 900 m elev., on tree branch; Transitional lowland forest, May 30, 2007, V.C. Linis 2690-07. Mt. Hibok-hibok, 650 m elev., on tree trunk; Residual lowland forest, May 31, 2007, V.C. Linis 2758-07. Mt. Hibok-hibok, 650 m elev., on tree trunk; Residual lowland forest, May 31, 2007, V.C. Linis 2760-07.

10. * Aerobryum speciosum Dozy \& Molk. - Luzon and Mindanao Mt. Timpoong, 1530 m elev., on tree branches; Montane forest, May 21, 2007, V.C. Linis 2592-07.

11. Barbula indica (Hook.) Spreng. - widespread

Sitio Campana, Brgy. Pandan, Mambajao (midslope of Mt. Timpoong), 520 m elev., on soil; Agricultural land, May 22, 2007, V.C. Linis 2609-07. Mt. Hibok-hibok, 850 m elev., on soil; Transitional lowland forest, May 31, 2007, V.C. Linis 2733-07.

12. Brachymenium nepalense Hook. - widespread

Brgy. Pandan, Mambajao (midslope of Mt. Timpoong), $800 \mathrm{~m}$ elev., on tree trunk; Lowland forest, May 19, 2007, V.C. Linis 2465-07. Danao, Mambajao (midslope of Mt. Timpoong), 1000 m elev., on tree trunk; Transitional lowland forest, May 19, 2007, V.C. Linis 2490-07. Mt. Hibokhibok, $650 \mathrm{~m}$ elev., on tree trunk; Secondary lowland forest, May 29, 2007, V.C. Linis 2658-07. Mt. Hibok-hibok, 860 m elev., on tree trunk; Transitional lowland forest, May 30, 2007, V.C. Linis 2683-07. 
13. *Bryum clavatum (Schimp.) Müll.Hal. - Luzon and Mindoro

Brgy. Tagdo, Mambajao (footslope of Mt. Hibok-hibok), $180 \mathrm{~m}$ elev., on boulder near hot spring; Human Settlement area, May 21, 2007, V.C. Linis 2613-07. Mt. Hibokhibok, 850 m elev., on boulder; Transitional lowland forest, May 31, 2007, V.C. 2737-07.

14. B. coronatum Schwägr. - widespread Mambajao Town proper, $10 \mathrm{~m}$ elev., on concrete wall; Agricultural land, May 19, 2007, V.C. Linis 2434-07. , Mt. Hibok-hibok, 180 m elev., on concrete wall; Agricultural land, May 29, 2007, V.C. Linis 2627-07., Mt. Hibok-hibok, 800 m elev., on stone wall; Transitional lowland forest, May 31, 2007, V.C. Linis 2750-07.

15. Callicostella papillata (Mont.) Mitt. - widespread

Danao, Mambajao (midslope of Mt. Timpoong), $1020 \mathrm{~m}$ elev., on wet rocks; Transitional lowland forest, May 20, 2007, V.C. Linis 2526-07. Mt. Hibok-hibok, $950 \mathrm{~m}$ elev., on rotten tree roots; Transitional lowland forest, May 30, 2007, V.C. Linis 2712-07. Mt. Hibok-hibok, 1000 m elev., on wet rocks; Transitional lowland forest, May 30, 2007, V.C. Linis 2719-07.

16. Calymperes graeffeanum Müll.Hal. - widespread

Mt. Hibok-hibok, 180 m elev., on dead tree trunk; Agricultural land, May 29, 2007, V.C. Linis 2625-07.

17. ${ }^{*}$ C. strictifolium (Mitt.) G.Roth. - Luzon and Mindanao

Brgy. Pandan, Mambajao, 680 m elev., on dead tree trunk; Lowland forest, May 19, 2007, V.C. Linis 2443-07. Mt. Hibok-hibok, 320 m elev., on dead tree trunk; Residual Lowland forest, May 29, 2007, V.C. Linis 2636-07. Mt. Hibok-hibok, 650 m elev., on dead tree trunk; Residual Lowland forest, May 31, 2007, V.C. Linis 2759-07.

18. C. tahitense (Sull.) Mitt. - widespread

Brgy. Pandan, Mambajao (midslope of Mt. Timpoong), $740 \mathrm{~m}$ elev., on lower tree trunk Lowland forest, May 19, 2007, V.C. Linis 2459-07. Mt. Hibok-hibok, 650 m elev., on lower tree trunk; Secondary Lowland forest, May 29, 2007, V.C. Linis 2652-07. Mt. Hibok-hibok, 600 m elev., on lower tree trunk; Residual Lowland forest, May 31, 2007, V.C. Linis 2775-07.

19. C. tenerum Müll.Hal. - widespread

Sitio Campana, Brgy. Pandan, Mambajao (midslope of Mt. Timpoong), $520 \mathrm{~m}$ elev., on dead tree trunk; Agricultural land, May 19, 2007, V.C. Linis 2432-07. Brgy. Tagdo, Mambajao (foot of Mt. Hibok-hibok), $180 \mathrm{~m}$ elev., on tree trunk near hot spring; Human Settlement area, May 21, 2007, V.C. Linis 2614-07. Mt. Hibok-hibok, 850 m elev., on tree trunk; Transitional Lowland forest, May 31, 2007, V.C. Linis 2738-07. Mt. Hibok-hibok, 800 m elev., on dead tree trunk; Transitional Lowland forest, May 31, 2007, V.C. Linis 2748-07.

20. *Calyptrochaeta fláccida (Broth.) Z.Iwats., B.C.Tan \& Touw - Luzon

Mt. Timpoong, $1250 \mathrm{~m}$ elev., on stem of small tree; Montane forest, May 21, 2007, V.C. Linis 2540-07. Mt. Timpoong, 1350 m elev., on dead tree trunk; Montane forest, May 21, 2007, V.C. Linis 2559-07. Mt. Timpoong, $1400 \mathrm{~m}$ elev., on tree branch; Montane forest, May 21, 2007, V.C. Linis 2574-07. Mt. Timpoong, 1530 m elev., on tree branch; Montane forest, May 21, 2007, V.C. Linis 2577-07.

21. ${ }^{*}$ C. remotifolia (Müll.Hal.) Z.Iwats., B.C Tan \& Touw - Luzon and Mindanao

Mt. Timpoong, $1250 \mathrm{~m}$ elev., on wet rocks; Montane forest, May 21, 2007, V.C. Linis 2537-07.

Danao, Mambajao, $1000 \mathrm{~m}$ elev., on wet rocks; Transitional Lowland forest, May 22, 2007, V.C. Linis 2601-07. Mt. Hibok-hibok, 1000 m elev., on wet rocks; Transitional Lowland forest, May 30, 2007, V.C. Linis 2725-07.

22. *Campylopodium medium (Duby) Giese \& J.-P.Frahm - Luzon and Mindoro Sitio Campana, Brgy. Pandan, Mambajao (midslope of Mt. Timpoong), $520 \mathrm{~m}$ elev., on eroded soil bank alongside road; Agricultural land, May 19, 2007, V.C. Linis 2426-07. Campana, Brgy. Pandan, Mambajao (midslope of Mt. Timpoong), $520 \mathrm{~m}$ elev., on eroded soil bank alongside road; Agricultural land, May 19, 2007, V.C. Linis 2436-07. Mt. Hibok-hibok, 180 m elev., on eroded soil bank alongside road; Agricultural land, May 29, 2007, V.C. Linis 2629-07. Mt. Hibokhibok, $800 \mathrm{~m}$ elev., on eroded soil bank alongside trail; Transitional Lowland forest, May 31, 2007, V.C. Linis 2752-07.

23. *Campylopus comosus (Schwägr.) Bosch \& Sande Lac. - Luzon and Mindanao Brgy. Pandan, Mambajao (midslope of Mt. Timpoong), 850 m elev., on trunk ofpalm (Caryota sp.) tree; Transitional Lowland forest, May 19, 2007, V.C. Linis 2475-07. 
24. C. umbellatus (Schwägr. \& Gaudich. ex Arn.) Paris - widespread

Mt. Hibok-hibok, 700 m elev., on moist soil bank; Transitional Lowland forest, May 29, 2007, V.C. Linis 2668-07.

25. Claopodium prionophyllum (Müll.Hal.) Broth. - widespread

Danao, Mambajao (midslope of Mt. Timpoong), $1000 \mathrm{~m}$ elev., on wet rocks along creek;

Transitional Lowland forest, May 20, 2007, V.C. Linis 2512-07. Danao, Mambajao (midslope of Mt. Timpoong), 1020 m elev., on wet rocks; Transitional Lowland forest, May 20, 2007, V.C. Linis 2531-07. Mt. Hibok-hibok, 950 m elev., on wet rocks; Transitional Lowland forest, May 30, 2007, V.C. Linis 2705-07.

26. * Clastobryum caudatum M.Fleisch. - Luzon and Mindoro

Danao, Mambajao (midslope of Mt. Timpoong), 1000 m elev., on dead tree trunk; Transitional Lowland forest, May 19, 2007, V.C. Linis 2499-07. Mt. Hibok-hibok, 900 m elev., on dead tree trunk; Transitional Lowland forest, May 30, 2007, V.C. Linis 2692-07.

27. ${ }^{*}$ Cryptopapillaria fuscescens (Hook.) M.Menzel - Luzon and Mindanao

Mt. Timpoong, 1400 m elev., on tree branch; Montane forest, May 21, 2007, V.C. Linis 2571-07.

28. *Cyathophorum hookerianum (Griff.) Mitt. - Luzon

Mt. Timpoong, $1350 \mathrm{~m}$ elev., on stem of small tree; Montane forest, May 21, 2007, V.C. Linis 2553-07.

29. ${ }^{\star}$ C. parvifolium Bosch \& Sande Lac. - Luzon and Mindanao

Mt. Timpoong, $1250 \mathrm{~m}$ elev., on tree trunk; Montane forest, May 21, 2007, V.C. Linis 2535-07.

Danao, Mambajao (midslope of Mt. Timpoong), $1020 \mathrm{~m}$ elev., on tree trunk; Transitional Lowland forest, May 20, 2007, V.C. Linis 2623-07. Mt. Hibok-hibok, 800 m elev., on tree trunk; Transitional Lowland forest, May 31, 2007, V.C. Linis 2746-07.

30. ${ }^{\star}$ C. spinosum (Müll.Hal.) M.Fleisch. - Luzon and some islands in the Visayas

Danao, Mambajao (midslope of Mt. Timpoong), $1000 \mathrm{~m}$ elev., on tree trunk; Transitional Lowland forest, May 19, 2007, V.C. Linis 2509-07. Danao, Mambajao (midslope of Mt. Timpoong), 1020 m elev., on wet rocks along trail; Transitional Lowland forest, May 20, 2007, V.C. Linis 2522-07. Mt. Hibok-hibok, 900 m elev., on tree trunk; Transitional Lowland forest, May 30, 2007, V.C. Linis 2702-07. Mt. Hibok-hibok, $1000 \mathrm{~m}$ elev., on wet rocks along trail; Transitional Lowland forest, May 30, 2007, V.C. Linis 2715-07.

31. Cyclodictyon blumeanum (Müll.Hal.) Kuntze - widespread Mt. Timpoong, 1530 m elev., on dead tree trunk; Montane forest, May 21, 2007, V.C. Linis 257907.

32. Dicranoloma assimile (Hampe) Paris - Luzon and Mindanao Mt. Hibok-hibok, 700 m elev., on tree trunk; Transitional Lowland forest, May 30, 2007, V.C. Linis 2671-07.

33. D. blumii (Nees) Ren. - widespread

Mt. Timpoong, 1530 m elev., on tree branch; Montane forest, May 21, 2007, V.C. Linis 2589-07.

34. D. braunii (Müll.Hal.) Paris - Luzon, Palawan, some islands in the Visayas and Mindanao

Danao, Mambajao (midslope of Mt. Timpoong), $1000 \mathrm{~m}$ elev., on tree trunk; Transitional Lowland forest, May 19, 2007, V.C. Linis 2478-07. Danao, Mambajao (midslope of Mt. Timpoong), 1000 m elev., on tree trunk; Transitional Lowland forest, May 19, 2007, V.C. Linis 2508-07. Mt. Hibokhibok, $900 \mathrm{~m}$ elev., on wet rocks along trail; Transitional Lowland forest, May 30, 2007, V.C. Linis 2700-07. Mt. Hibok-hibok, 900 m elev., on tree trunk; Transitional Lowland forest, May 30, 2007, V.C. Linis 2701-07.

35. ${ }^{\star} D$. reflexum (Müll.Hal.) Renauld. - Luzon and some islands in the Visayas

Brgy. Pandan, Mambajao (midslope of Mt. Timpoong), $850 \mathrm{~m}$ elev., on tree branch; Transitional Lowland forest, May 19, 2007, V.C. Linis 2471-07. Danao, Mambajao (midslope of Mt. Timpoong), 1000 m elev., on tree trunk; Transitional Lowland forest, May 19, 2007, V.C. Linis 2502-07. Mt. Timpoong, $1350 \mathrm{~m}$ elev., on tree trunk; Montane forest, May 21, 2007, V.C. Linis 2552-07. Mt. Hibok-hibok, $700 \mathrm{~m}$ elev., on tree branch; Transitional Lowland forest, May 29, 2007, V.C. Linis 2664-07. Mt. Hibok-hibok, 900 m elev., on tree trunk; Transitional Lowland forest, May 30, 2007, V.C. Linis 2695-07.

36. ${ }^{\star}$ Diphyscium rupestre Dozy \& Molk. - Luzon and some islands in the Visayas Danao, Mambajao (midslope of Mt. Timpoong), $1020 \mathrm{~m}$ elev., on wet rocks along trail; 
Transitional Lowland forest, May 20, 2007, V.C. Linis 2532-07.

37. *Distichophyllum cuspidatum (Dozy \& Molk.) Dozy \& Molk. - Luzon and Mindanao

Mt. Timpoong, 1530 m elev., on tree branch; Montane forest, May 21, 2007, V.C. Linis 2580-07.

38. $\quad{ }^{\star}$ D. mittenii Bosch \& Sande Lac. - Luzon and Mindanao

Mt. Timpoong, 1530 m elev., on tree trunk; Montane forest, May 21, 2007, V.C. Linis 2576-07.

39. $* D$. nigricaule Mitt. ex Bosch \& Sande Lac. - Luzon, Mindoro, Palawan and Western Mindanao Mt. Timpoong, 1350 m elev., on wet rocks; Montane forest, May 21, 2007, V.C. Linis 2548-07.

40. ${ }^{\star} D$. osterwaldii M.Fleisch. - Luzon and some islands in the Visayas

Mt. Timpoong, 1250 m elev., on wet rocks; Montane forest, May 21, 2007, V.C. Linis 2536-07. Mt. Timpoong, $1530 \mathrm{~m}$ elev., on base of tree trunk; Montane forest, May 21, 2007, V.C. Linis 2597-07.

41. Ectropotheciella distichophylla (Hampe ex Dozy \& Molk.) M.Fleisch. - widespread Mt. Hibok-hibok, 650 m elev., on tree trunk; Residual Lowland forest, May 29, 2007, V.C. Linis 2657-07.

42. Ectropothecium buitenzorgii (Bel.) Mitt. - widespread Mt. Hibok-hibok, 900 m elev., on base of decaying tree trunk; Transitional Lowland forest, May 30, 2007, V.C. Linis 2689-07.

43. E. dealbatum (Reinw. \& Hornsch.) A.Jaeger - widespread

Mt. Hibok-hibok, 320 m elev., on moist soil beside trail; Residual Lowland forest, May 29, 2007, V.C. Linis 2634-07.

44. ${ }^{\star}$ E. falciforme (Dozy \& Molk.) A.Jaeger - Luzon and Mindanao

Mt. Timpoong, 1350 m elev., on dead tree trunk; Montane forest, May 21, 2007, V.C. Linis 255607. Mt. Timpoong, $1350 \mathrm{~m}$ elev., on stem of small tree; Montane forest, May 21, 2007, V.C. Linis 2557-07.

45. E. monumentorum (Duby) A.Jaeger - widespread. Mt. Hibok-hibok, 320 m elev., on rocks; Residual Lowland forest, May 29, 2007, V.C. Linis 263707.

46. ${ }^{\star}$ E. perminutum Broth. ex E.B.Bartram - Luzon and Mindoro

Danao, Mambajao (midslope of Mt. Timpoong), 1020 m elev., on wet rocks; Transitional Lowland forest, May 20, 2007, V.C. Linis 2525-07. Mt. Hibok-hibok, 1000 m elev., on wet rocks; Transitional Lowland forest, May 30, 2007, V.C. Linis 2718-07.

47. Erythrodontium julaceum (Hook ex Schwägr.) Paris - widespread Mt. Hibok-hibok, 510 m elev., on tree trunk; Secondary Lowland forest, May 29, 2007, V.C. Linis 2651-07.

48. ${ }^{\star}$ Exostratum blumii (Nees ex Hampe) L.T.Ellis - Luzon and Mindanao

Danao, Mambajao (midslope of Mt. Timpoong), 1000 m elev., on base of tree trunk; Transitional Lowland forest, May 19, 2007, V.C. Linis 2495-07. Mt. Hibok-hibok, 900 m elev., on base of tree trunk; Transitional Lowland forest, May 30, 2007, V.C. Linis 2688-07.

49. Fissidens crenulatus Mitt. var. elmeri (Broth.) Z.Iwats. \& Tad.Suzuki - widespread Mt. Timpoong, 1250 m elev., on trunk of tree ferns; Montane forest, May 21, 2007, V.C. Linis 2539-07.

50. F. crispulus Brid. - widespread

Brgy. Tagdo, Mambajao (footslope of Mt. Hibok-hibok), $180 \mathrm{~m}$ elev., on soil adjacent to hot srping; Human Settlement area, May 21, 2007, V.C. Linis 2616-07. Danao, Mambajao (midslope of Mt. Timpoong), 1000 m elev., on wet rocks near creek; Transitional Lowland forest, May 21, 2007, V.C. Linis 2620-07. Mt. Hibok-hibok, 850 m elev., on soil; Transitional Lowland forest, May 31, 2007, V.C. Linis 2740-07. Mt. Hibok-hibok, 850 m elev., on wet rocks; Transitional Lowland forest, May 31, 2007, V.C. Linis 2743-07.

51. F. nobilis Griff. - widespread

Brgy. Pandan, Mambajao (midslope of Mt. Timpoong), 740 m elev., on soil bank alongside trail; Lowland forest, May 19, 2007, V.C. Linis 2460-07. Danao, Mambajao (midslope of Mt. Timpoong), 1000 m elev., on wet rocks near creek; Transitional Lowland forest, May 20, 2007, V.C. 
Linis 2515-07. Danao, Mambajao (midslope of Mt. Timpoong), 1000 m elev., on wet rocks along trail; Transitional Lowland forest, May 22, 2007, V.C. Linis 2600-07. Mt. Hibok-hibok, 650 m elev., on soil bank alongside trail; Secondary Lowland forest, May 29, 2007, V.C. Linis 2653-07.

Mt. Hibok-hibok, 600 m elev., on soil bank alongside trail; Residual Lowland forest, May 31, 2007, V.C. Linis 2777-07.

52. ${ }^{\star}$ F. oblongifolius Hook.f. \& Wilson - Mindoro and some islands in the Visayas

Mt. Hibok-hibok, 950 m elev., on wet rocks; Transitional Lowland forest, May 30, 2007, V.C. Linis 2708-07.

53. *F. punctulatus Sande Lac. - Luzon

Mambajao Town Proper, 10 m elev., on rocks; Human Settlement area, May 21, 2007, V.C. Linis 2611-07. Mt. Hibok-hibok, 850 m elev., on rocks; Transitional Lowland forest, May 31, 2007, V.C. Linis 2735-07.

54. Floribundaria floribunda (Dozy \& Molk.) M.Fleisch. - widespread

Brgy. Pandan, Mambajao (midslope of Mt. Timpoong), 720 m elev., on lower tree trunk; Lowland forest, May 19, 2007, V.C. Linis 2448-07. Mt. Timpoong, 1350 m elev., on tree branch; Montane forest, May 21, 2007, V.C. Linis 2558-07. Brgy. Pandan, Mambajao (midslope of Mt. Timpoong), 800 m elev., on tree trunk; Lowland forest, May 22, 2007, V.C. Linis 2598-07. Mt. Hibok-hibok, 320 m elev., on lower tree trunk; Residual Lowland forest, May 29, 2007, V.C. Linis 2641-07. Mt. Hibok-hibok, 650 m elev., on lower tree trunk; Residual Lowland forest, May 31, 2007, V.C. Linis 2764-07.

55. Funaria hygrometrica Hedw. - widespread Mt. Hibok-hibok, 1000 m elev., on burnt soil and charred wood; Transitional Lowland forest, May 30, 2007, V.C. Linis 2724-07.

56. * Garckea comosa (Dozy \& Molk.) Wijk \& Margad. - Luzon, Mindoro and Palawan Sitio Campana, Brgy. Pandan, Mambajao (midslope of Mt. Timpoong), $520 \mathrm{~m}$ elev., on eroded soil bank alongside road; Agricultural land, May 19, 2007, V.C. Linis 2427-07.

57. *Garovaglia angustifolia Mitt. - Luzon and Mindanao

Brgy. Pandan, Mambajao (midslope of Mt. Timpoong), $680 \mathrm{~m}$ elev., on tree trunk and branches; Lowland forest, May 19, 2007, V.C. Linis 2447-07. Brgy. Pandan, Mambajao (midslope of Mt. Timpoong), 850 m elev., on tree branch; Transitional Lowland forest, May 19, 2007, V.C. Linis 2472-07.Danao, Mambajao (midslope of Mt. Timpoong), 1000 m elev., on tree branch; Transitional Lowland forest, May 19, 2007, V.C. Linis 2482-07. Mt. Timpoong, $1250 \mathrm{~m}$ elev., on tree trunk; Montane forest, May 21, 2007, V.C. Linis 2533-07. Mt. Hibok-hibok, 320 m elev., on tree trunk and branches; Residual Lowland forest, May 29, 2007, V.C. Linis 2460-07.

Mt. Hibok-hibok, 700 m elev., on tree branch; Transitional Lowland forest, May 29, 2007, V.C. Linis 2665-07. Mt. Hibok-hibok, 860 m elev., on tree branch; Transitional Lowland forest, May 30, 2007, V.C. Linis 2675-07. Mt. Hibok-hibok, 650 m elev., on tree trunk and branches; Residual Lowland forest, May 31, 2007, V.C. Linis 2763-07.

58. G. elegans (Dozy \& Molk.) Hampe ex Bosch \& Sande Lac. - widespread

Brgy. Pandan, Mambajao (midslope of Mt. Timpoong), 720 m elev., on tree trunk; Lowland forest, May 19, 2007, V.C. Linis 2452-07. Brgy. Pandan, Mambajao (midslope of Mt. Timpoong), 740 m elev., on tree trunk; Transitional Lowland forest, May 19, 2007, V.C. Linis 2457-07. Brgy. Pandan, Mambajao (midslope of Mt. Timpoong), 850 m elev., on tree branch; Transitional Lowland forest, May 19, 2007, V.C. Linis 2476-07. Danao, Mambajao (midslope of Mt. Timpoong), 1000 m elev., on tree trunk; Transitional Lowland forest, May 19, 2007, V.C. Linis 2483-07. Danao, Mambajao (midslope of Mt. Timpoong), 1000 m elev., on tree trunk; Transitional Lowland forest, May 19, 2007, V.C. Linis 2506-07. Mt. Timpoong, 1350 m elev., on tree trunk; Montane forest, May 21, 2007, V.C. Linis 2544-07. Mt. Hibok-hibok, 450 m elev., on tree trunk; Secondary Lowland forest, May 29, 2007, V.C. Linis 2645-07. Mt. Hibok-hibok, 510 m elev., on tree trunk; Secondary Lowland forest, May 29, 2007, V.C. Linis 2650-07. Mt. Hibokhibok, 700 m elev., on tree trunk; Transitional Lowland forest, May 29, 2007, V.C. Linis 2669-07. Mt. Hibok-hibok, 860 m elev., on tree trunk; Transitional Lowland forest, May 30, 2007, V.C. Linis 2676-07. Mt. Hibok-hibok, 900 m elev., on tree trunk; Transitional Lowland forest, May 30, 2007, V.C. Linis 2699-07. Mt. Hibok-hibok, 650 m elev., on tree trunk; Residual Lowland forest, May 31, 2007, V.C. Linis 2768-07. Mt. Hibok-hibok, 600 m elev., on tree trunk; Residual Lowland forest, May 31, 2007, V.C. Linis 2773-07. 
59. *Glossadelphus hermaphroditus M.Fleisch. - Mindanao

Mt. Timpoong, 1530 m elev., on base of tree trunk; Montane forest, May 21, 2007, V.C. Linis 2585-07.

60. Himantocladium cyclophyllum (Müll.Hal.) M.Fleisch. - widespread

Brgy. Pandan, Mambajao (midslope of Mt. Timpoong), 680 m elev., on tree branch; Lowland forest, May 19, 2007, V.C. Linis 2466-07. Brgy. Pandan, Mambajao (midslope of Mt. Timpoong), 800 m elev., on tree trunk; Lowland forest, May 19, 2007, V.C. Linis 2468-07. Danao, Mambajao (midslope of Mt. Timpoong), 1000 m elev., on tree stem near creek; Transitional Lowland forest, May 20, 2007, V.C. Linis 2511-07. Danao, Mambajao (midslope of Mt. Timpoong), 1000 m elev., on tree trunk near creek; Transitional Lowland forest, May 20, 2007, V.C. Linis 2518-07. Danao, Mambajao (midslope of Mt. Timpoong), $1020 \mathrm{~m}$ elev., on tree trunk; Transitional Lowland forest, May 20, 2007, V.C. Linis 2529-07. Hibok-hibok, 320 m elev., on tree branch; Residual Lowland forest, May 29, 2007, V.C. Linis 2639-07. Mt. Hibok-hibok, 650 m elev., on tree trunk; Secondary Lowland forest, May 29, 2007, V.C. Linis 2661-07. Mt. Hibok-hibok, 950 m elev., on tree stem near creek; Transitional Lowland forest, May 30, 2007, V.C. Linis 2711-07. Mt. Hibok-hibok, 950 m elev., on tree trunk near creek; Transitional Lowland forest, May 30, 2007, V.C. Linis 2699-07. Mt. Hibok-hibok, 1000 m elev., on tree trunk; Transitional Lowland forest, May 30, 2007, V.C. Linis 2722-07. Mt. Hibok-hibok, 650 m elev., on tree branch; Residual Lowland forest, May 31, 2007, V.C. Linis 2762-07.

61. Homaliodendron flabellatum (Sm.) M.Fleisch. - widespread

Danao, Mambajao (midslope of Mt. Timpoong), 1000 m elev., on tree trunk; Transitional Lowland forest, May 19, 2007, V.C. Linis 2477-07. Danao, Mambajao (midslope of Mt. Timpoong), 1000 m elev., on tree trunk; Transitional Lowland forest, May 19, 2007, V.C. Linis 2481-07.

Mt. Timpoong, 1350 m elev., on tree trunk; Montane forest, May 21, 2007, V.C. Linis 2547-07.

Mt. Timpoong, 1400 m elev., on tree trunk; Montane forest, May 21, 2007, V.C. Linis 2570-07.

Mt. Hibok-hibok, 700 m elev., on tree trunk; Transitional Lowland forest, May 29, 2007, V.C. Linis 2670-07. Mt. Hibok-hibok, 700 m elev., on tree trunk; Transitional Lowland forest, May 30, 2007, V.C. Linis 2674-07.

62. Hyophila involuta (Hook.) A.Jaeger. - widespread

Mambajao Town Proper, 10 m elev., on concrete wall; Human Settlement area, May 21, 2007, V.C. Linis 2610-07. Mt. Hibok-hibok, 850 m elev., on rock wall; Transitional Lowland forest, May 31, 2007, V.C. Linis 2734-07.

63. *Hypnodendron milnei Mitt. ssp. korthalsii (Bosch \& Sande Lac. ex Paris) Touw - Luzon, Mindoro, Palawan and Western Mindanao

Danao, Mambajao (midslope of Mt. Timpoong), $1000 \mathrm{~m}$ elev., on wet rocks along trail; Transitional Lowland forest, May 19, 2007, V.C. Linis 2485-07. Danao, Mambajao (midslope of Mt. Timpoong), $1000 \mathrm{~m}$ elev., on wet rocks along trail; Transitional Lowland forest, May 19, 2007, V.C. Linis 2504-07. Mt. Hibok-hibok, $860 \mathrm{~m}$ elev., on wet rocks along trail; Transitional Lowland forest, May 30, 2007, V.C. Linis 2678-07. Mt. Hibok-hibok, 900 m elev., on wet rocks alongside trail; Transitional Lowland forest, May 30, 2007, V.C. Linis 2697-07.

64. H. reinwardtii (Schwägr.) Lindb. ex A.Jaeger \& Sauerb. ssp. caducifolium (Herzog) Touw widespread

Danao, Mambajao (midslope of Mt. Timpoong), 1000 m elev., on base of tree trunk; Transitional Lowland forest, May 19, 2007, V.C. Linis 2496-07. Mt. Timpoong, 1250 m elev., on tree trunk; Montane forest, May 21, 2007, V.C. Linis 2534-07. Mt. Timpoong, 1400 m elev., on tree trunk; Montane forest, May 21, 2007, V.C. Linis 2572-07. Mt. Timpoong, $1530 \mathrm{~m}$ elev., on tree trunk; Montane forest, May 21, 2007, V.C. Linis 2591-07.

65. ${ }^{*} H$. vitiense Mitt. - Luzon and Mindanao

Danao, Mambajao (midslope of Mt. Timpoong), 1020 m elev., on wet boulders; Transitional Lowland forest, May 20, 2007, V.C. Linis 2532-07. Danao, Mambajao (midslope of Mt. Timpoong), 1000 m elev., on tree trunk; Transitional Lowland forest, May 22, 2007, V.C. Linis 2602-07. Mt. Hibok-hibok, 950 m elev., on dead tree branch; Transitional Lowland forest, May 30, 2007, V.C. Linis 2713-07. Mt. Hibok-hibok, 1000 m elev., on wet boulders; Transitional Lowland forest, May 30, 2007, V.C. Linis 2716-07. Mt. Hibok-hibok, 1000 m elev., on tree trunk; Transitional Lowland forest, May 30, 2007, V.C. Linis 2726-07.

66. *Isopterygium albescens (Hook.) A.Jaeger - Luzon and some islands in the Visayas Mt. Timpoong, $1250 \mathrm{~m}$ elev., on stem of small tree; Montane forest, May 21, 2007, V.C. Linis 253807. Mt. Timpoong, 1530 m elev., on tree branch; Montane forest, May 21, 2007, V.C. Linis 2595-07. 
67. *I. minutirameum (Müll.Hal.) A.Jaeger - Luzon, Mindoro and Palawan

Mt. Timpoong, 1350 m elev., on woody vine; Montane forest, May 21, 2007, V.C. Linis 2550-07.

68. * Leucobryum aduncum Dozy \& Molk. - Luzon, Mindoro and Palawan

Sitio Campana, Brgy. Pandan, Mambajao (midslope of Mt. Timpoong), $520 \mathrm{~m}$ elev., on tree trunk alongside road; Agricultural land, May 19, 2007, V.C. Linis 2428-07.

69. * ${ }^{\star}$. chlorophyllosum Müll.Hal. - Mindoro and some islands in the Visayas

Danao, Mambajao (midslope of Mt. Timpoong), $1000 \mathrm{~m}$ elev., on base of tree trunk; Transitional Lowland forest, May 19, 2007, V.C. Linis 2505-07. Mt. Hibok-hibok, 900 m elev., on base of tree trunk; Transitional Lowland forest, May 30, 2007, V.C. Linis 2698-07.

70. L. javense (Brid.) Mitt. - widespread

Danao, Mambajao (midslope of Mt. Timpoong), $1000 \mathrm{~m}$ elev., on base of tree trunk; Transitional Lowland forest, May 19, 2007, V.C. Linis 2487-07. Mt. Timpoong, 1530 m elev., on tree branch; Montane forest, May 21, 2007, V.C. Linis 2581-07. Mt. Hibok-hibok, $860 \mathrm{~m}$ elev., on base of tree trunk; Transitional Lowland forest, May 30, 2007, V.C. Linis 2680-07.

71. ${ }^{*}$ L. juniperoideum (Brid.) Müll.Hal. - Luzon, Mindoro and Mindanao

Brgy. Pandan, Mambajao (midslope of Mt. Timpoong), 800 m elev., on tree branch; Grassland, May 19, 2007, V.C. Linis 2464-07.

72. L. sanctum (Nees ex Schwägr.) Hampe - widespread

Danao, Mambajao (midslope of Mt. Timpoong), $1000 \mathrm{~m}$ elev., on dead tree branch; Transitional Lowland forest, May 20, 2007, V.C. Linis 2520-07.

73. L. scalare Müll.Hal. ex M.Fleisch. - Luzon and some islands in the Visayas Mt. Timpoong, $1530 \mathrm{~m}$ elev., on dead tree trunk; Montane forest, May 21, 2007, V.C. Linis 258207.

74. * Leucoloma molle (Müll.Hal.) Mitt. - Luzon and Mindanao

Danao, Mambajao (midslope of Mt. Timpoong), 1000 m elev., on tree trunk; Transitional Lowland forest, May 19, 2007, V.C. Linis 2488-07. Mt. Hibok-hibok, 860 m elev., on tree trunk; Transitional Lowland forest, May 30, 2007, V.C. Linis 2681-07.

75. * Leucophanes angustifolium Renauld \& Cardot - Luzon and Mindanao Brgy. Pandan, Mambajao (midslope of Mt. Timpoong), 720 m elev., on lower tree trunk; Lowland forest, May 19, 2007, V.C. Linis 2453-07. Mt. Timpoong, $1250 \mathrm{~m}$ elev., on trunk of tree ferns; Montane forest, May 21, 2007, V.C. Linis 2542-07. Mt. Hibok-hibok, $450 \mathrm{~m}$ elev., on lower tree trunk; Secondary Lowland forest, May 29, 2007, V.C. Linis 2646-07. Mt. Hibok-hibok, 600 m elev., on lower tree trunk; Residual Lowland forest, May 31, 2007, V.C. Linis 2769-07.

76. L. candidum (Schwägr.) Lindb. - widespread

Brgy. Pandan, Mambajao (midslope of Mt. Timpoong), 850 m elev., on trunk of palm (Caryota sp.) tree; Transitional Lowland forest, May 22, 2007, V.C. Linis 2607-07. Mt. Hibok-hibok, 850 m elev., on trunk of palm tree; Transitional Lowland forest, May 31, 2007, V.C. Linis 2731-07.

77. * L. glaucum (Schwägr.) Mitt. - Luzon and Mindanao

Brgy. Tagdo, Mambajao (footslope of Mt. Hibok-hibok), $180 \mathrm{~m}$ elev., on base of palm's trunk near to hot spring; Human Settlement area, May 21, 2007, V.C. Linis 2612-07. Mt. Hibok-hibok, 850 m elev., on base of palm's trunk; Transitional Lowland forest, May 31, 2007, V.C. Linis 2736-07.

78. * L. octoblepharioides Brid. - Luzon and Mindanao

Sitio Campana, Brgy. Pandan, Mambajao (midslope of Mt. Timpoong), $520 \mathrm{~m}$ elev., on base of tree trunk; Agricultural land, May 19, 2007, V.C. Linis 2431-07. Brgy. Pandan, Mambajao (midslope of Mt. Timpoong), 720 m elev., on tree trunk; Lowland forest, May 19, 2007, V.C. Linis 2455-07. Mt. Hibok-hibok, 180 m elev., on base of tree trunk; Agricultural land, May 29, 2007, V.C. Linis 2624-07. Mt. Hibok-hibok, $510 \mathrm{~m}$ elev., on tree trunk; Secondary Lowland forest, May 29, 2007, V.C. Linis 2648-07. Mt. Hibok-hibok, $800 \mathrm{~m}$ elev., on base of tree trunk; Transitional Lowland forest, May 31, 2007, V.C. Linis 2747-07. Mt. Hibok-hibok, 600 m elev., on tree trunk; Residual Lowland forest, May 31, 2007, V.C. Linis 2771-07.

79. Lopidium struthiopteris (Brid.) M.Fleisch. - widespread Danao, Mambajao (midslope of Mt. Timpoong), $1000 \mathrm{~m}$ elev., on tree trunk; Transitional Lowland forest, May 20, 2007, V.C. Linis 2484-07. Danao, Mambajao (midslope of Mt. Timpoong), 1000 m elev., on tree trunk; Transitional Lowland forest, May 20, 2007, V.C. Linis 2492-07. Danao, Mambajao (midslope of Mt. Timpoong), 1020 m elev., on tree trunk; Transitional Lowland forest, 
May 20, 2007, V.C. Linis 2521-07. Mt. Hibok-hibok, 860 m elev., on tree trunk; Transitional Lowland forest, May 31, 2007, V.C. Linis 2677-07. Mt. Hibok-hibok, 900 m elev., on tree trunk; Transitional Lowland forest, May 31, 2007, V.C. Linis 2685-07. Mt. Hibok-hibok, 950 m elev., on tree trunk; Transitional Lowland forest, May 31, 2007, V.C. Linis 2714-07.

80. *Macromitrium angustifolium Dozy \& Molk. - Luzon and Mindoro Danao, Mambajao (midslope of Mt. Timpoong), 1020 m elev., on tree branch near creek; Transitional Lowland forest, May 20, 2007, V.C. Linis 2514-07. Mt. Hibok-hibok, 950 m elev., on tree branch; Transitional Lowland forest, May 30, 2007, V.C. Linis 2707-07.

81. M. orthostichum Nees \& Schwägr. - Luzon, Mindoro, Palawan and Western Mindanao Brgy. Pandan, Mambajao (midslope of Mt. Timpoong), $800 \mathrm{~m}$ elev., on tree branch; Grassland, May 19, 2007, V.C. Linis 2461-07. Mt. Hibok-hibok, 650 m elev., on tree branch; Grassland, May 29, 2007, V.C. Linis 2654-07. Mt. Hibok-hibok, 500 m elev., on tree branch; Grassland, May 31, 2007, V.C. Linis 2778-07.

82. M. salakanum Müll.Hal. - Luzon, Mindoro, Palawan and Western Mindanao Brgy. Pandan, Mambajao (midslope of Mt. Timpoong), $800 \mathrm{~m}$ elev., on tree trunk; Grassland, May 19, 2007, V.C. Linis 2462-07. Brgy. Pandan, Mambajao (midslope of Mt. Timpoong), 800 m elev., on tree branches; Grassland, May 19, 2007, V.C. Linis 2463-07. Danao, Mambajao (midslope of Mt. Timpoong), 1000 m elev., on tree branch; Transitional Lowland forest, May 22, 2007, V.C. Linis 2603-07. Mt. Hibok-hibok, 650 m elev., on tree trunk; Grassland, May 29, 2007, V.C. Linis 2655-07. Mt. Hibok-hibok, 1000 m elev., on tree branch; Transitional Lowland forest, May 30, 2007, V.C. Linis 2727-07.

83. ${ }^{\star} M$. subtile Schwägr. ssp. subuligerum (Bosch \& Sande Lac.) M.Fleisch. - Luzon, Mindoro, Palawan and Western Mindanao

Brgy. Pandan, Mambajao (midslope of Mt. Timpoong), $680 \mathrm{~m}$ elev., on tree branch; Lowland forest, May 19, 2007, V.C. Linis 2439-07. Mt. Hibok-hibok, $180 \mathrm{~m}$ elev., on tree branch; Residual Lowland forest, May 29, 2007, V.C. Linis 2632-07. Mt. Hibok-hibok, $650 \mathrm{~m}$ elev., on tree branch; Residual Lowland forest, May 31, 2007, V.C. Linis 2755-07.

84. *Meiothecium hamatum (Müll.Hal.) Broth. - Luzon and some islands in the Visayas Danao, Mambajao (midslope of Mt. Timpoong), 1000 m elev., on tree trunk; Transitional Lowland forest, May 19, 2007, V.C. Linis 2479-07.

85. Meteorium polytrichum Dozy \& Molk. - widespread Brgy. Pandan, Mambajao (midslope of Mt. Timpoong), $800 \mathrm{~m}$ elev., on tree branch; Lowland forest, May 19, 2007, V.C. Linis 2469-07. Mt. Hibok-hibok, 700 m elev., on tree branch; Secondary Lowland forest, May 29, 2007, V.C. Linis 2662-07.

86. Neckeropsis lepineana (Mont.) M.Fleisch. - widespread Mt. Timpoong, 1400 m elev., on tree trunk; Montane forest, May 21, 2007, V.C. Linis 2573-07.

87. Octoblepharum albidum Hedw. - widespread Mt. Hibok-hibok, 650 m elev., on tree branches; Grassland, May 29, 2007, V.C. Linis 2656-07.

88. * Orthomnion javense (M.Fleisch.) T.J.Kop. - Mindanao

Brgy. Pandan, Mambajao (midslope of Mt. Timpoong), $680 \mathrm{~m}$ elev., on tree trunk; Lowland forest, May 19, 2007, V.C. Linis 2438-07. Mt. Hibok-hibok, 180 m elev., on tree trunk; Residual Lowland forest, May 29, 2007, V.C. Linis 2631-07. Mt. Hibok-hibok, 650 m elev., on tree trunk; Residual Lowland forest, May 31, 2007, V.C. Linis 2754-07.

89. *O. loheri Broth. - Luzon

Brgy. Pandan, Mambajao (midslope of Mt. Timpoong), $800 \mathrm{~m}$ elev., on tree trunk; Lowland forest, May 19, 2007, V.C. Linis 2466-07. Mt. Hibok-hibok, 650 m elev., on tree trunk; Secondary Lowland forest, May 29, 2007, V.C. Linis 2659-07.

90. *Pelekium investe (Mitt.) Touw - Luzon and Mindanao

Brgy. Tagdo, Mambajao (footslope of Mt. Hibok-hibok), $180 \mathrm{~m}$ elev., on soil near hot spring; Human Settlement area, May 21, 2007, V.C. Linis 2615-07. Mt. Hibok-hibok, 850 m elev., on soil; Transitional Lowland forest, May 31, 2007, V.C. Linis 2739-07.

91. *P. synoicum (Touw) Touw - Luzon and Mindanao

Brgy. Tagdo, Mambajao (footslope of Mt. Hibok-hibok), $200 \mathrm{~m}$ elev., on rock near hot spring; Human Settlement area, May 21, 2007, V.C. Linis 2617-07. Mt. Hibok-hibok, 850 m elev., on rock; Transitional Lowland forest, May 31, 2007, V.C. Linis 2741-07. 
92. P. velatum Mitt. - widespread

Mt. Hibok-hibok, 900 m elev., on lower tree trunk; Transitional Lowland forest, May 30, 2007, V.C. Linis 2693-07.

93. Philonotis bartramioides (Griff.) D. G.Griffin \& W.R.Buck - Luzon and Mindanao

Sitio Campana, Brgy. Pandan, Mambajao (midslope of Mt. Timpoong), 520 m elev., on eroded soil bank; Agricultural land, May 19, 2007, V.C. Linis 2433-07. Mt. Hibok-hibok, 180 m elev., on eroded soil bank; Agricultural land, May 29, 2007, V.C. Linis 2626-07. Mt. Hibok-hibok, 800 m elev., on eroded soil bank; Secondary Lowland forest, May 31, 2007, V.C. Linis 2749-07.

94. *P. hastata (Duby) Wijk \& Margad. - Luzon and some islands in the Visayas

Sitio Campana, Brgy. Pandan, Mambajao (midslope of Mt. Timpoong), 520 m elev., on soil; Agricultural land, May 22, 2007, V.C. Linis 2608-07.

95. P. mollis (Duby \& Molk.) Mitt. - Luzon

Mt. Hibok-hibok, 850 m elev., on soil; Transitional Lowland forest, May 31, 2007, V.C. Linis 273207.

96. * Pogonatum camusii (Thér.) Touw - Luzon and Mindanao

Mt. Timpoong, 1350 m elev., on exposed soil bank near creek; Montane forest, May 21, 2007, V.C. Linis 2621-07.

97. *P. microphyllum (Dozy \& Molk.) Dozy \& Molk. - Luzon and some islands in the Visayas Mt. Hibok-hibok, 850 m elev., on exposed soil bank; Transitional Lowland forest, May 31, 2007, V.C. Linis 2744-07.

98. P. neesii (Müll.Hal.) Dozy - widespread

Sitio Campana, Brgy. Pandan, Mambajao (midslope of Mt. Timpoong), 680 m elev., on soil bank alongside trail; Lowland forest, May 19, 2007, V.C. Linis 2437-07. Brgy. Pandan, Mambajao (midslope of Mt. Timpoong), $720 \mathrm{~m}$ elev., on soil bank alongside of trail; Lowland forest, May 19, 2007, V.C. Linis 2454-07. Mt. Timpoong, $1350 \mathrm{~m}$ elev., on exposed soil bank near creek; Montane forest, May 21, 2007, V.C. Linis 2543-07. Mt. Hibok-hibok, $180 \mathrm{~m}$ elev., on soil bank alongside trail; Lowland forest, May 29, 2007, V.C. Linis 2630-07. Mt. Hibok-hibok, 510 m elev., on soil bank alongside trail; Secondary Lowland forest, May 29, 2007, V.C. Linis 2647-07. Mt. Hibokhibok, 650 m elev., on soil bank alongside trail; Residual Lowland forest, May 31, 2007, V.C. Linis 2753-07. Mt. Hibok-hibok, $600 \mathrm{~m}$ elev., on soil bank alongside of trail; Residual Lowland forest, May 31, 2007, V.C. Linis 2770-07.

99. Pseudoleskeopsis zippellii (Dozy \& Molk.) Broth. - Luzon Mt. Hibok-hibok, $800 \mathrm{~m}$ elev., on tree trunk near creek; Transitional Lowland forest, May 31, 2007, V.C. Linis 2745-07.

100. *Pterobryopsis crassicaulis (Müll.Hal.) M.Fleisch. - Luzon and some islands in the Visayas Brgy. Pandan, Mambajao (midslope of Mt. Timpoong), 720 m elev., on lower tree trunk; Lowland forest, May 19, 2007, V.C. Linis 2449-07. Brgy. Pandan, Mambajao (midslope of Mt. Timpoong), 850 m elev., on tree branch; Transitional Lowland forest, May 19, 2007, V.C. Linis 2470-07. Danao, Mambajao (midslope of Mt. Timpoong), $1000 \mathrm{~m}$ elev., on tree trunk; Transitional Lowland forest, May 19, 2007, V.C. Linis 2480-07. Danao, Mambajao (midslope of Mt. Timpoong), $1000 \mathrm{~m}$ elev., on tree trunk; Transitional Lowland forest, May 19, 2007, V.C. Linis 2489-07. Mt. Hibok-hibok, 450 m elev., on lower tree trunk; Secondary Lowland forest, May 29, 2007, V.C. Linis 2462-07. Mt. Hibok-hibok, $700 \mathrm{~m}$ elev., on tree branch; Transitional Lowland forest, May 29, 2007, V.C. Linis 2663-07. Mt. Hibok-hibok, $700 \mathrm{~m}$ elev., on tree trunk; Transitional Lowland forest, May 30, 2007, V.C. Linis 2673-07. Mt. Hibok-hibok, $860 \mathrm{~m}$ elev., on tree trunk; Transitional Lowland forest, May 30, 2007, V.C. Linis 2682-07. Mt. Hibok-hibok, 650 m elev., on lower tree trunk; Residual Lowland forest, May 31, 2007, V.C. Linis 2765-07.

101. *P. gedehensis M.Fleisch. - Luzon and Mindanao

Brgy. Pandan, Mambajao (midslope of Mt. Timpoong), $680 \mathrm{~m}$ elev., on dead tree trunk; Lowland forest, May 19, 2007, V.C. Linis 2445-07. Mt. Hibok-hibok, 320 m elev., on dead tree trunk; Residual Lowland forest, May 29, 2007, V.C. Linis 2638-07. Mt. Hibok-hibok, 650 m elev., on dead tree trunk; Residual Lowland forest, May 31, 2007, V.C. Linis 2761-07.

102. Pyrrhobryum spiniforme (Hedw.) Mitt. - widespread Danao, Mambajao (midslope of Mt. Timpoong), $1000 \mathrm{~m}$ elev., on dead tree trunk; Transitional Lowland forest, May 19, 2007, V.C. Linis 2503-07. Mt. Timpoong, $1530 \mathrm{~m}$ elev., on dead tree trunk; Montane forest, May 21, 2007, V.C. Linis 2584-07. Danao, Mambajao (midslope of Mt. 
Timpoong), 1000 m elev., on base of tree trunk; Transitional Lowland forest, May 22, 2007, V.C. Linis 2605-07. Mt. Hibok-hibok, 900 m elev., on dead tree trunk; Transitional Lowland forest, May 30, 2007, V.C. Linis 2696-07. Mt. Hibok-hibok, 850 m elev., on base of tree trunk; Transitional Lowland forest, May 30, 2007, V.C. Linis 2729-07.

103. *Racopilum cuspidigerum (Schwägr.) Ångström - Luzon and some islands in the Visayas Sitio Campana, Brgy. Pandan, Mambajao (midslope of Mt. Timpoong), 520 m elev., on base of tree trunk; Agricultural land, May 19, 2007, V.C. Linis 2435-07. Brgy. Pandan, Mambajao (midslope of Mt. Timpoong), 740 m elev., on tree root; Lowland forest, May 19, 2007, V.C. Linis 2458-07. Mt. Hibok-hibok, 180 m elev., on base of tree trunk; Agricultural land, May 29, 2007, V.C. Linis 2628-07. Mt. Hibok-hibok, 600 m elev., on tree root; Residual Lowland forest, May 31, 2007, V.C. Linis 2774-07.

104. Racopilum spectabile Reinw. \& Hornsch. - widespread

Mt. Hibok-hibok, 800 m elev., on base of tree trunk; Transitional Lowland forest, May 31, 2007, V.C. Linis 2751-07.

105. Radulina hamata (Dozy \& Molk.) W.R.Buck \& B.C.Tan - widespread

Mt. Timpoong, 1250 m elev., on dead tree trunk; Montane forest, May 21, 2007, V.C. Linis 2541 07. Mt. Timpoong, $1350 \mathrm{~m}$ elev., on stem of small tree; Montane forest, May 21, 2007, V.C. Linis 2546-07. Mt. Timpoong, 1350 m elev., on dead tree trunk; Montane forest, May 21, 2007, V.C. Linis 2549-07. Mt. Timpoong, 1350 m elev., on dead tree trunk; Montane forest, May 21, 2007, V.C. Linis 2564-07. Mt. Timpoong, 1350 m elev., on tree branch; Montane forest, May 21, 2007, V.C. Linis 2569-07. Mt. Timpoong, 1530 m elev., on dead tree trunk; Montane forest, May 21, 2007, V.C. Linis 2587-07.

106. Sematophyllum microcladioides (Broth.) Broth. - Luzon and Mindanao Mt. Hibok-hibok, 700 m elev., on tree trunk; Transitional Lowland forest, May 30, 2007, V.C. Linis 2672-07.

107. Spiridens reinwardtii Nees - widespread

Danao, Mambajao (midslope of Mt. Timpoong), 1000 m elev., on trunk of tree ferns; Transitional Lowland forest, May 19, 2007, V.C. Linis 2486-07. Mt. Timpoong, $1200 \mathrm{~m}$ elev., on trunk of tree fern; Montane forest, May 22, 2007, V.C. Linis 2604-07. Danao, Mambajao (midslope of Mt. Timpoong), 1000 m elev., on trunk of tree ferns; Transitional Lowland forest, May 22, 2007, V.C. Linis 2606-07. Mt. Hibok-hibok, $860 \mathrm{~m}$ elev., on trunk of tree ferns; Transitional Lowland forest, May 30, 2007, V.C. Linis 2679-07. Mt. Hibok-hibok, 1000 m elev., on trunk of tree fern; Montane forest, May 30, 2007, V.C. Linis 2728-07. Mt. Hibok-hibok, 850 m elev., on trunk of tree ferns; Transitional Lowland forest, May 31, 2007, V.C. Linis 2730-07.

108. * Symphysodon neckeroides Dozy \& Molk. - Luzon, Mindoro, Palawan and Western Mindanao Brgy. Pandan, Mambajao (midslope of Mt. Timpoong), 720 m elev., on tree trunk; Lowland forest, May 19, 2007, V.C. Linis 2451-07. Brgy. Pandan, Mambajao (midslope of Mt. Timpoong), 800 m elev., on tree trunk; Lowland forest, May 19, 2007, V.C. Linis 2467-07. Brgy. Pandan, Mambajao (midslope of Mt. Timpoong), $850 \mathrm{~m}$ elev., on tree branch; Transitional Lowland forest, May 19, 2007, V.C. Linis 2473-07. Mt. Hibok-hibok, 450 m elev., on tree trunk; Secondary Lowland forest, May 29, 2007, V.C. Linis 2644-07. Mt. Hibok-hibok, 650 m elev., on tree trunk; Secondary Lowland forest, May 29, 2007, V.C. Linis 2660-07. Mt. Hibok-hibok, 700 m elev., on tree branch; Transitional Lowland forest, May 29, 2007, V.C. Linis 2666-07. Mt. Hibok-hibok, 650 m elev., on tree trunk; Residual Lowland forest, May 31, 2007, V.C. Linis 2767-07.

109. *Symphysodontella attenuatula M.Fleisch. - Luzon and Mindanao Brgy. Pandan, Mambajao (midslope of Mt. Timpoong), 720 m elev., on tree trunk; Lowland forest, May 19, 2007, V.C. Linis 2450-07. Mt. Hibok-hibok, 450 m elev., on tree trunk; Secondary Lowland forest, May 29, 2007, V.C. Linis 2643-07. Mt. Hibok-hibok, 650 m elev., on tree trunk; Residual Lowland forest, May 31, 2007, V.C. Linis 2766-07.

110. *S. subulata Broth. - Luzon and Mindoro

Brgy. Pandan, Mambajao (midslope of Mt. Timpoong), $680 \mathrm{~m}$ elev., on tree trunk; Lowland forest, May 19, 2007, V.C. Linis 2440-07. Brgy. Pandan, Mambajao (midslope of Mt. Timpoong), 680 m elev., on tree trunk; Lowland forest, May 19, 2007, V.C. Linis 2441-07. Danao, Mambajao (midslope of Mt. Timpoong), 1000 m elev., on tree trunk; Transitional Lowland forest, May 19, 2007, V.C. Linis 2501-07. Mt. Hibok-hibok, 320 m elev., on tree trunk; Residual Lowland forest, May 29, 2007, V.C. Linis 2633-07. Mt. Hibok-hibok, 900 m elev., on tree trunk; Transitional Lowland forest, May 30, 2007, V.C. Linis 2694-07. Mt. Hibok-hibok, 650 m elev., on tree trunk; 
Residual Lowland forest, May 31, 2007, V.C. Linis 2756-07. Mt. Hibok-hibok, 650 m elev., on tree trunk; Residual Lowland forest, May 31, 2007, V.C. Linis 2757-07.

111. *Syrrhopodon prolifer Schwägr. - Mindanao

Danao, Mambajao (midslope of Mt. Timpoong), 1020 m elev., on lower tree trunk; Transitional Lowland forest, May 20, 2007, V.C. Linis 2528-07. Mt. Hibok-hibok, 1000 m elev., on lower tree trunk; Transitional Lowland forest, May 30, 2007, V.C. Linis 2721-07.

112. *S. spiculosus Hook. \& Grev. - Mindoro and some islands in the Visayas

Mt. Timpoong, 1350 m elev., on tree trunk; Montane forest, May 21, 2007, V.C. Linis 2567-07.

113. S. tristichus Nees ex Schwägr. - widespread

Mt. Timpoong, 1350 m elev., on tree trunk; Montane forest, May 21, 2007, V.C. Linis 2566-07.

Mt. Timpoong, 1530 m elev., on tree branch; Montane forest, May 21, 2007, V.C. Linis 2583-07.

Mt. Timpoong, 1530 m elev., on tree trunk; Montane forest, May 21, 2007, V.C. Linis 2588-07.

Mt. Timpoong, 1530 m elev., on tree branch; Montane forest, May 21, 2007, V.C. Linis 2594-07.

114. *Taxithelium alare Broth. - Luzon and some islands in the Visayas

Danao, Mambajao (midslope of Mt. Timpoong), $1000 \mathrm{~m}$ elev., on tree branch; Transitional Lowland forest, May 19, 2007, V.C. Linis 2491-07. Mt. Hibok-hibok, 900 m elev., on tree branch; Transitional Lowland forest, May 30, 2007, V.C. Linis 2684-07.

115. T. instratum (Brid.) Broth. - widespread

Danao, Mambajao (midslope of Mt. Timpoong), 1020 m elev., on dead tree trunk; Transitional Lowland forest, May 20, 2007, V.C. Linis 2530-07.

116. * T. nepalense (Schwägr.) Broth. - Luzon and Mindanao

Brgy. Tagdo, Mambajao (footslope of Mt. Hibok-hibok), 180 m elev., on rock near hot spring; Human Settlement area, May 21, 2007, V.C. Linis 2618-07. Mt. Hibok-hibok, 850 m elev., on rock; Transitional Lowland forest, May 31, 2007, V.C. Linis 2742-07.

117. T. vernieri (Duby) Besch. - widespread

Mt. Timpoong, 1350 m elev., on tree branch; Montane forest, May 21, 2007, V.C. Linis 2561-07.

Mt. Hibok-hibok, 1000 m elev., on dead tree trunk; Transitional Lowland forest, May 30, 2007, V.C. Linis 2723-07.

118. Thuidium cymbifolium (Dozy \& Molk.) Dozy \& Molk. - widespread

Danao, Mambajao (midslope of Mt. Timpoong), 1000 m elev., on dead tree trunk; Transitional Lowland forest, May 20, 2007, V.C. Linis 2510-07. Danao, Mambajao (midslope of Mt.

Timpoong), 1000 m elev., on lower tree trunk; Transitional Lowland forest, May 20, 2007, V.C. Linis 2516-07. Mt. Hibok-hibok, 900 m elev., on dead tree trunk; Transitional Lowland forest, May 30, 2007, V.C. Linis 2703-07. Mt. Hibok-hibok, 950 m elev., on lower tree trunk; Transitional Lowland forest, May 30, 2007, V.C. Linis 2709-07.

119. *T. plumulosum (Dozy \& Molk.) Dozy \& Molk. - Luzon and Mindanao Danao, Mambajao (midslope of Mt. Timpoong), 1000 m elev., on lower tree trunk; Transitional Lowland forest, May 19, 2007, V.C. Linis 2500-07. Danao, Mambajao (midslope of Mt. Timpoong), 1000 m elev., on rocks near creek; Transitional Lowland forest, May 20, 2007, V.C. Linis 2513-07. Mt. Hibok-hibok, 950 m elev., on rocks near creek; Transitional Lowland forest, May 30, 2007, V.C. Linis 2706-07.

120. ${ }^{*}$ T. pristocalyx (Müll.Hal.) A.Jaeger var. samoanum (Mitt.) Touw - Luzon and Mindanao Brgy. Pandan, Mambajao (midslope of Mt. Timpoong), 720 m elev., on tree roots; Lowland forest, May 19, 2007, V.C. Linis 2456-07. Danao, Mambajao (midslope of Mt. Timpoong), 1000 m elev., on base of tree trunk; Transitional Lowland forest, May 19, 2007, V.C. Linis 2494-07. Mt. Hibok-hibok, 510 m elev., on tree roots; Secondary Lowland forest, May 29, 2007, V.C. Linis 2649-07. Mt. Hibok-hibok, 900 m elev., on base of tree trunk; Transitional Lowland forest, May 30, 2007, V.C. Linis 2687-07. Mt. Hibok-hibok, 600 m elev., on tree roots; Residual Lowland forest, May 31, 2007, V.C. Linis 2772-07.

121. *Trachythecium micropyxis (Broth.) E.B.Bartram - Luzon and Mindanao Danao, Mambajao (midslope of Mt. Timpoong), 1020 m elev., on wet rocks; Transitional Lowland forest, May 20, 2007, V.C. Linis 2527-07. Mt. Hibok-hibok, 1000 m elev., on wet rocks; Transitional Lowland forest, May 30, 2007, V.C. Linis 2720-07. 
122. T. verrucosum (A.Jaeger) M.Fleisch. - widespread

Mt. Timpoong, 1350 m elev., on tree trunk; Montane forest, May 21, 2007, V.C. Linis 2551-07.

123. *Trematodon longicollis Michx. - Luzon and Mindoro

Sitio Campana, Brgy. Pandan, Mambajao (midslope of Mt. Timpoong), 520 m elev., on soil bank alongside road; Agricultural land, May 19, 2007, V.C. Linis 2429-07.

124. *Trichosteleum stigmosum Mitt. - Luzon, Mindoro and Mindanao

Danao, Mambajao (midslope of Mt. Timpoong), $1020 \mathrm{~m}$ elev., on dead tree trunk; Transitional Lowland forest, May 20, 2007, V.C. Linis 2524-07. Mt. Hibok-hibok, 1000 m elev., on dead tree trunk; Transitional Lowland forest, May 30, 2007, V.C. Linis 2717-07.

125. * Trismegistia calderensis (Sull.) Broth. - Luzon and Mindanao

Danao, Mambajao (midslope of Mt. Timpoong), 1000 m elev., on lower tree trunk; Transitional Lowland forest, May 20, 2007, V.C. Linis 2517-07. Mt. Timpoong, $1350 \mathrm{~m}$ elev., on dead tree trunk; Montane forest, May 21, 2007, V.C. Linis 2545-07. Mt. Timpoong, $1350 \mathrm{~m}$ elev., on tree trunk; Montane forest, May 21, 2007, V.C. Linis 2555-07. Mt. Timpoong, $1350 \mathrm{~m}$ elev., on dead tree trunk; Montane forest, May 21, 2007, V.C. Linis 2565-07. Mt. Timpoong, $1530 \mathrm{~m}$ elev., on trunk of dead tree; Montane forest, May 21, 2007, V.C. Linis 2596-07. Mt. Hibok-hibok, 950 m elev., on lower tree trunk; Transitional Lowland forest, May 30, 2007, V.C. Linis 2710-07.

126. T. rigida (Mitt.) Broth. - widespread

Mt. Timpoong, $1530 \mathrm{~m}$ elev., on tree branch; Montane forest, May 21, 2007, V.C. Linis 2575-07.

Mt. Timpoong, 1530 m elev., on tree trunk; Montane forest, May 21, 2007, V.C. Linis 2590-07.

127. Vesicularia montagnei (Schimp.) Broth. - widespread

Brgy. Tagdo, Mambajao (footslope of Mt. Hibok-hibok), 180 m elev., on wet soil and rocks near hot spring; Human Settlement area, May 21, 2007, V.C. Linis 2619-07.

128. V. reticulata (Dozy \& Molk.) Broth. - widespread

Mt. Hibok-hibok, 600 m elev., on moist soil and rocks beside trail; Transitional Lowland forest, May 31, 2007, V.C. Linis 2776-07.

129. *Warburgiella breviseta (Broth.) Broth. - Luzon and Mindanao

Mt. Timpoong, 1350 m elev., on dead tree trunk; Montane forest, May 21, 2007, V.C. Linis 256207. 